\title{
Be STARS IN THE OPEN CLUSTER NGC 6830
}

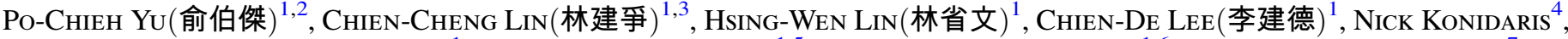 \\ Chow-Choong NGeOw(饒兆聰 $)^{1}$, Wing-Huen IP(葉永烜 $)^{1,5}$, Wen-PING Chen $(\text { 陳文屏 })^{1,6}$, HuI-CHEN CHEN $(\text { 陳慧真 })^{7}$, \\ Matthew A. Malkan ${ }^{2}$, Chan-Kao Chang(章展誥 $)^{1}$, Russ Laher(良主嶺亞 $)^{8}$, Li-Ching Huang $(\text { 黃立晴 })^{1}$, \\ Yu-Chi Cheng(鄭宇棋) ${ }^{1}$, Rick Edelson ${ }^{9}$, Andreas RitTer $^{1,10}$, Robert Quimby ${ }^{11}$, Sagi Ben-Ami ${ }^{12}$, Eran. O. OfeK ${ }^{12}$, \\ Jason Surace ${ }^{8}$, AND Shrinivas R. KulKarni ${ }^{13}$ \\ ${ }^{1}$ Graduate Institute of Astronomy, National Central University, 300 Jhongda Road, Jhongli 32001, Taiwan \\ ${ }_{2}^{2}$ Department of Physics and Astronomy, University of California, Los Angeles, CA 90024, USA \\ ${ }^{3}$ Key Laboratory for Research in Galaxies and Cosmology, Shanghai Astronomical Observatory, \\ Chinese Academy of Sciences, 80 Nandan Road Shanghai 200030, China
${ }^{4}$ Cahill Center for Astronomy and Astrophysics, California Institute of Technology, 1200 E. California Boulevard, Pasadena, CA 91125, USA \\ ${ }^{5}$ Institute of Space Science, National Central University, 300 Jhongda Road, Jhongli 32001, Taiwan \\ ${ }^{6}$ Department of Physics, National Central University, 300 Jhongda Road, Jhongli 32001, Taiwan \\ ${ }^{7}$ Department of Natural Sciences and Sustainable Development, Ministry of Science and Technology, 106, Sec. 2, Heping E. Road, Taipei 10622, Taiwan \\ ${ }^{8}$ Spitzer Science Center, California Institute of Technology, M/S 314-6, Pasadena, CA 91125, USA \\ ${ }^{9}$ Department of Astronomy, University of Maryland, College Park, MD 20742, USA \\ ${ }^{10}$ Department of Astrophysical Sciences, Princeton University, Princeton, NJ 08544, USA \\ ${ }^{11}$ Kavli-Institute for the Physics and Mathematics of the Universe, University of Tokyo, Kashiwanoha 5-1-5, Kashiwa-shi, Chiba, Japan \\ ${ }^{12}$ Department of Particle Physics and Astrophysics, The Weizmann Institute of Science, Rehovot 76100, Israel \\ ${ }^{13}$ Division of Physics, Mathematics and Astronomy, California Institute of Technology, Pasadena, CA 91125, USA \\ Received 2015 November 11; accepted 2016 February 24; published 2016 April 20
}

\begin{abstract}
We report the discovery of two new Be stars, and re-identify one known Be star in the open cluster NGC 6830. Eleven $\mathrm{H} \alpha$ emitters were discovered using the $\mathrm{H} \alpha$ imaging photometry of the Palomar Transient Factory Survey. Stellar membership of the candidates was verified with photometric and kinematic information using 2MASS data and proper motions. The spectroscopic confirmation was carried out by using the Shane $3 \mathrm{~m}$ telescope at the Lick observatory. Based on their spectral types, three $\mathrm{H} \alpha$ emitters were confirmed as $\mathrm{Be}$ stars with $\mathrm{H} \alpha$ equivalent widths greater than $-10 \AA$. Two objects were also observed by the new spectrograph spectral energy distribution-machine (SED-machine) on the Palomar 60-inch Telescope. The SED-machine results show strong H $\alpha$ emission lines, which are consistent with the results of the Lick observations. The high efficiency of the SED-machine can provide rapid observations for Be stars in a comprehensive survey in the future.
\end{abstract}

Key words: galaxies: star clusters: individual (NGC 6830) - instrumentation: spectrographs - stars: emissionline, Be

\section{INTRODUCTION}

Be stars are non-supergiant B-type stars with Balmer emission lines, especially the $\mathrm{H} \alpha$ lines. Be stars are also characterized by their fast rotation, color excess at infrared wavelengths, and continuum/emission-line variability (Koubský et al. 1997; Hubert \& Floquet 1998; Townsend et al. 2004). Some Be stars show the variability with periods that might be due to pulsation or rotation (Porter \& Rivinius 2003; Rivinius et al. 2003). Previous studies have found the enhancement of $\mathrm{Be}$ phenomena in young clusters (Wisniewski \& Bjorkman 2006; Mathew et al. 2008), while McSwain \& Gies (2005) showed an overall increase in Be frequency with age until 100 Myr. McSwain \& Gies (2005) also indicated that $\mathrm{Be}$ star formation is not strongly related to the cluster density. Furthermore, the Be star fraction in open clusters seems to be increasing with lower metallicity; between $17.5 \%$ and $40 \%$ of B stars were found to be Be stars in the Large Magellanic Cloud and Small Magellanic Cloud (SMC; see a detailed review in Rivinius et al. 2013).

It is well accepted that the $\mathrm{H} \alpha$ emissions of $\mathrm{Be}$ stars originate from the circumstellar material, which is suggested to be a flattened disk (Quirrenbach et al. 1994, 1997; Wood et al. 1997; Carciofi \& Bjorkman 2006). The disks are found to be in Keplerian rotation and stable (Kraus et al. 2012; Meilland et al. 2012). The material might be moved from the stellar surface to form the circumstellar disk (e.g., the decretion disk) through the combination of fast rotation and other mechanisms, such as nonradial pulsations or magnetic fields (Lee et al. 1991; Porter \& Rivinius 2003; Townsend et al. 2004; Carciofi et al. 2009). There are several possible origins of the fast rotating star: (1) binary interaction (Pols et al. 1991), by which, during the interaction process, mass and angular momentum can be transferred from the primary star to the mass gainer star, and the latter might be spun-up to a fast rotating star, (2) they might be born as fast rotators, and (3) they have been spun-up during the main-sequence evolution.

To further understand the nature of Be stars, several surveys of Be stars have been carried out in the past (Mclaughlin 1932; Jaschek et al. 1964; Drew et al. 2005; Chojnowski et al. 2015; Lin et al. 2015; Raddi et al. 2015). Because stars in clusters share age and metallicity, Be stars in a sample of clusters with different ages play an essential role in the study of their evolution. Previous studies have searched for emission-line objects in a number of star clusters in the SMC and MW. (Fabregat \& Torrejón 2000; Keller et al. 2001; Wisniewski \& Bjorkman 2006; Mathew et al. 2008; Martayan et al. 2010; Mathew \& Subramaniam 2011). Particularly, McSwain \& Gies (2005) provided the most extensive survey of Be stars in the MW; they investigated 55 clusters and concluded that $73 \%$ of the rapid rotators might be spun-up by the effect of mass 

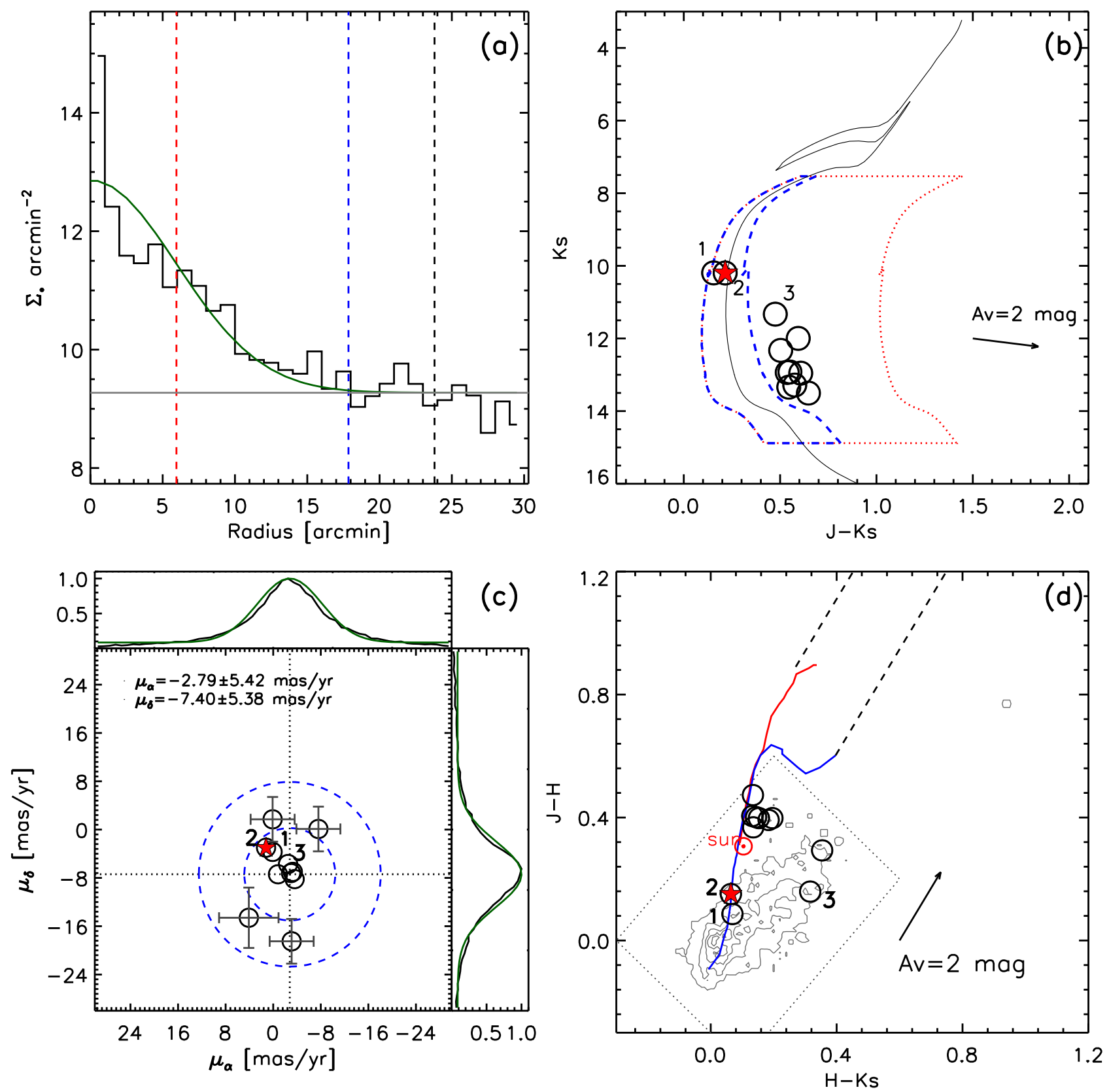

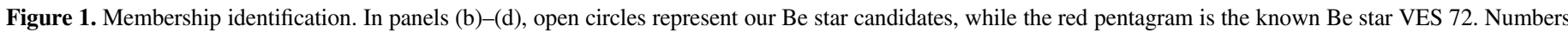

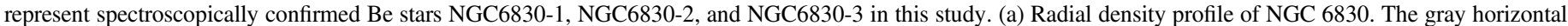

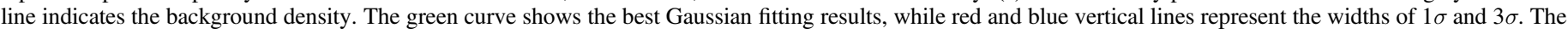

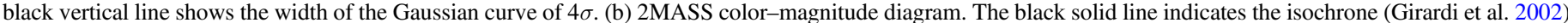

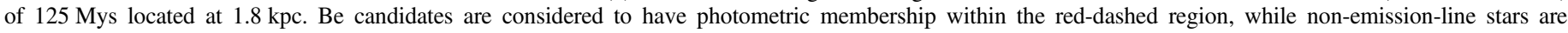

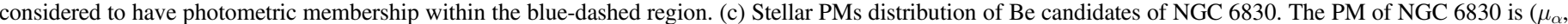

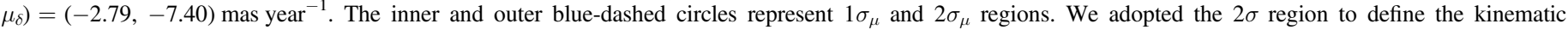

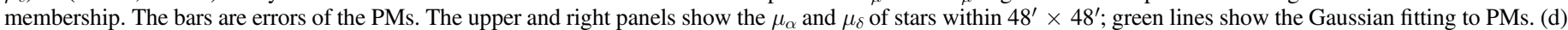

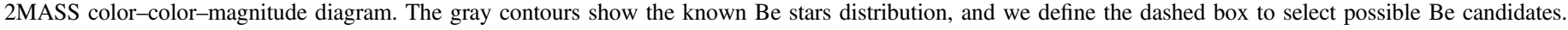

transfer between binaries. Moreover, McSwain et al. (2009) also showed that the distributions of the projected rotational velocity of Be stars and normal B-type stars are significantly different, indicating that they might be different stellar populations, and Be stars cannot be drawn from a sample of normal B stars. Theoretical simulations also imply that most Be stars could be formed during the phase of binary interactions (de Mink et al. 2013; Shao \& Li 2014). These studies further supported a spin-up scheme for the evolutionary sequence of Be stars.
Nevertheless, the sample of Be stars in open clusters is far from complete due to a couple of difficulties: (1) a comprehensive spectroscopic survey is time consuming and (2) spectroscopic surveys are often limited to bright stars. Therefore, with the wide field of view (7.3 square degree) of the 48-inch Samuel Oschin Telescope, the Palomar Transient Factory (PTF; Law et al. 2009) project provides an efficient way to search for emission-line candidates in open clusters using $\mathrm{H} \alpha$ imaging photometry (Yu et al. 2015). Furthermore, a new instrument called the spectral energy distribution-machine 


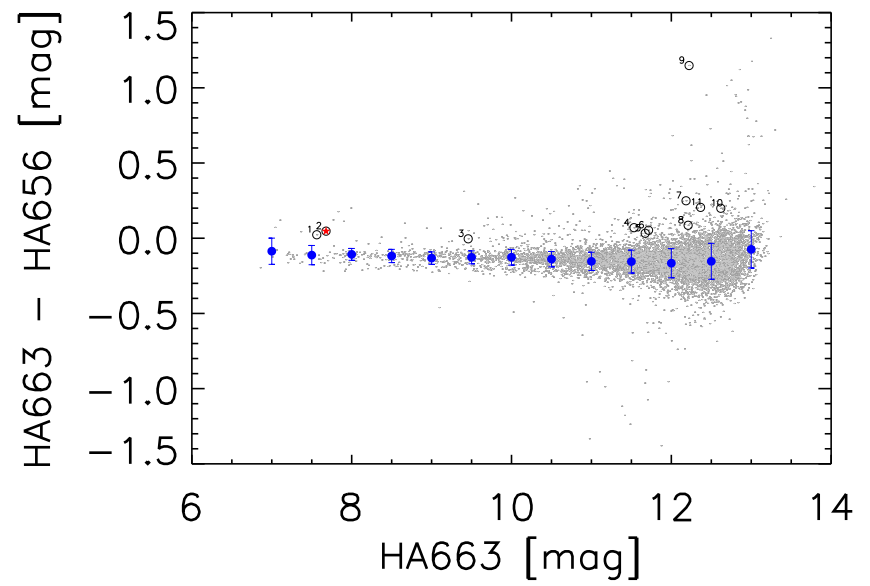

Figure 2. $\mathrm{H} \alpha$ emitter candidates selection. Gray dots: stars used for calculating photometric scattering; open circles: emission-line candidates with membership confirmation; numbers represent Be candidates NGC6830-1-NGC6830-11; red pentagram: known Be stars VES 72. Blue filled circles represent the mean values of HA663-HA656 within each $0.5 \mathrm{mag}$ bin. The error bars represent the photometric scattering $\sigma_{p}$, which are calculated with the error propagated from photometric and systematic errors. Stars with HA663-HA656 > mean $+2 \sigma_{p}$ are selected as emission-line candidates.

(SED-machine; Ben-Ami et al. 2012; Ngeow et al. 2013; Ritter et al. 2014) provides a possible solution for the fast and efficient follow-up spectroscopic observations for the emissionline candidates found in PTF, because the highly efficient SEDmachine is designed to classify transient events rapidly with a low-resolution $(\lambda / \delta \lambda \sim 100)$ integrated field unit (IFU) spectrograph that covers a large wavelength range $(370-920 \mathrm{~nm})$. Thus, a comprehensive survey of Be stars in star clusters can be accomplished with a combination of the PTF $\mathrm{H} \alpha$ imaging photometry and the SED-machine.

Here we present the results of searching for Be stars in an open cluster NGC $6830 \quad\left(\alpha_{2000}=19^{\mathrm{h}} 50^{\mathrm{m}} 59^{\mathrm{s}}, \delta_{2000}=23^{\circ} 06^{\prime} 00^{\prime \prime}\right)$. NGC 6830, located at a distance of $1.8 \mathrm{kpc}$ (Kharchenko et al. 2013) in the constellation Vulpecula (Barkhatova 1957), has a fairly loose structure. In contrast to our previous work (NGC 663 with an age of $31 \mathrm{Myr}$, Yu et al. 2015), the age of NGC 6830 is about $125 \mathrm{Myr}$ (Kharchenko et al. 2013) and only one Be star has been reported in this cluster (Hoag \& Applequist 1965) prior to our work. Using the relation of effective temperature and age, Peña et al. (2011) determined that the age of NGC 6830 is $\log$ (age) $=7.69$ years $(48.9 \mathrm{Myr}$ ) with the hottest star $(17,000 \mathrm{~K})$ in the membership of 19 stars. It seems that the age given by Kharchenko et al. (2013) is not consistent with that of Peña et al. (2011). However, while the membership in Peña et al. (2011) is only determined from the distance, Kharchenko et al. (2013) included the proper motions (PMs) to determine the membership and give the log (age) $=8.105 \pm 0.062$ years $(125 \mathrm{Myr})$. Furthermore, the range of the effective temperature for the membership in Peña et al. (2011) is from 12,000 to $17,000 \mathrm{~K}$, indicating that the $\log ($ age $)$ is between 7.69 and 8.22 years, which is also consistent with the age given by Kharchenko et al. (2013). Thus, here we adopted the age given by Kharchenko et al. (2013).

In addition to the age difference, we selected NGC 6830 as the subject of this work because the known Be star is fairly bright and hence it is suitable to be observed with the SEDmachine during its commissioning runs. Therefore, the main goal of this work is to demonstrate the capability of the combined PTF $\mathrm{H} \alpha$ imaging photometry, the archival 2MASS and proper motions data, and the SED-machine in the search of Be stars in open clusters. This will lay the foundation of our future work in this area. In Section 2, we describe the methodology of identifying emission-line stars and the determination of cluster membership using the PTF $\mathrm{H} \alpha$ imaging photometry. In Sections 3 and 4, we describe our spectroscopic observations and present our results based on these observations. Section 5 gives a summary and discussion of this study.

\section{IDENTIFICATION OF Be CANDIDATES AND MEMBERSHIP}

The methodology of searching for Be star candidates and identifying membership has been presented in our pilot project. Further details can be found in Yu et al. (2015) and will not be repeated here. Briefly, procedures that we followed in this study are summarized below.

1. Determining the searching region based on the radial density profile. The stars were selected from the PPMXL data set with a signal-to-noise ratio $(\mathrm{S} / \mathrm{N}) \geqslant 10$ in 2MASS $J, H$, and $K_{s}$ bands. The half-Gaussian fitting gives a $3 \sigma$ radius of 17 !85 (Figure 1(a)). Because open clusters have irregular shapes, we therefore adopted a box with the side of $48^{\prime}(4 \sigma)$ as our searching region for NGC 6830 to cover possible candidates.

2. Applying the $\mathrm{H} \alpha$ imaging photometry to identify possible $\mathrm{H} \alpha$ emitters. The $\mathrm{H} \alpha$ emitters should have a more significant flux excess in the on-line image than in the off-line image, where the on-line and off-line images were taken through the HA 656 and HA 663 narrow-band filters. These images were processed for bias corrections, flat-fielding, and astrometric calibration with pipelines developed at the Infrared Processing and Analysis Center (IPAC; Laher et al. 2014). Following the methods and criteria as given in $\mathrm{Yu}$ et al. (2015), we selected the $\mathrm{H} \alpha$ emitter candidates that stand out in the plot of the differential HA663-HA656 flux as possible $\mathrm{H} \alpha$ emitters (see Figure 2).

3. Verifying the photometric membership using near-infrared data. The near-infrared $J_{-}, H$-, and $K_{s}$-band data are obtained from the 2MASS point source catalog (Cutri et al. 2003). Possible photometric members of NGC 6830 are determined by selecting stars within the region that are near the isochrone in the 2MASS $K_{s}$ versus $J-K_{s}$ color-magnitude diagram (Figure 1(b)). Considering that Be stars might exhibit a large infrared excess (Lee \& Chen 2011), we extended the selection region to $J-K_{s}$ $\sim 1.2$ mag (Figure 1(b)) for the $\mathrm{H} \alpha$ emitter candidates.

4. Verifying the kinematic membership using the PMs. Because about $10 \%$ of objects in PPMXL data include spurious entries (Röser et al. 2010), Kharchenko et al. (2012) have averaged their PMs and computed errors. Thus, we used PMs provided by Kharchenko et al. (2012) to calculate the averaged $\mathrm{PM}\left(\mu_{\alpha}, \mu_{\delta}\right)$ and standard deviations $\left(\sigma_{\mu \alpha}, \sigma_{\mu \delta}\right)$ of stars within the $47 ! 6 \times 47 ! 6$ region by fitting a Gaussian distribution to the PMs. The adopted $\sigma_{\mu}$ included the error propagated from $\sigma_{\mu \alpha}$ and $\sigma_{\mu \delta}$. Stars were then considered as the kinematic membership if their PMs are within the $2 \sigma_{\mu}$ region (Figure 1(c)). 
Table 1

Be Candidates

\begin{tabular}{|c|c|c|c|c|c|c|c|c|c|c|}
\hline$\overline{\mathrm{ID}}$ & $\begin{array}{c}\text { R.A. } \\
\text { (hh:mm:ss) }\end{array}$ & $\begin{array}{c}\text { Decl. } \\
\text { (hh:mm:ss) }\end{array}$ & $\begin{array}{c}\mu_{\alpha} \\
\left(\operatorname{mas~yr}^{-1}\right)\end{array}$ & $\begin{array}{c}\mu_{\delta} \\
\left(\operatorname{mas~yr}^{-1}\right)\end{array}$ & $\begin{array}{c}\text { epm } \\
\left(\text { mas yr }^{-1}\right)\end{array}$ & $\begin{array}{c}K_{s} \\
(\mathrm{mag})\end{array}$ & $\begin{array}{l}J-H \\
(\mathrm{mag})\end{array}$ & $\begin{array}{c}H-K_{s} \\
(\mathrm{mag})\end{array}$ & $\begin{array}{c}\text { HA663 } \\
\text { (mag) }\end{array}$ & $\begin{array}{c}\text { HA663-HA656 } \\
\text { (mag) }\end{array}$ \\
\hline NGC 6830-1 & 19:51:00.47 & $+23: 06: 50.6$ & 0.10 & -3.70 & 1.50 & 10.350 & 0.086 & 0.069 & 7.563 & 0.023 \\
\hline NGC 6830-2 & $19: 50: 58.55$ & $+23: 08: 28.4$ & 1.20 & -3.00 & 1.50 & 10.412 & 0.151 & 0.064 & 7.679 & 0.047 \\
\hline NGC 6830-3 & $19: 51: 58.13$ & $+22: 44: 59.8$ & -3.30 & -7.00 & 3.70 & 11.806 & 0.159 & 0.316 & 9.458 & -0.004 \\
\hline NGC 6830-4 & 19:50:02.31 & $+23: 00: 15.2$ & -3.60 & -8.20 & 3.70 & 12.831 & 0.368 & 0.135 & 11.533 & 0.070 \\
\hline NGC 6830-5 & $19: 50: 05.02$ & $+23: 02: 55.2$ & -2.50 & -5.70 & 3.70 & 12.592 & 0.398 & 0.196 & 11.673 & 0.033 \\
\hline NGC 6830-6 & $19: 50: 04.61$ & $+23: 20: 27.3$ & -7.60 & 0.10 & 3.70 & 13.473 & 0.405 & 0.132 & 11.715 & 0.053 \\
\hline NGC 6830-7 & $19: 52: 08.58$ & $+22: 59: 59.8$ & 4.10 & -14.60 & 5.00 & 14.151 & 0.293 & 0.354 & 12.184 & 0.249 \\
\hline NGC 6830-8 & $19: 50: 02.94$ & $+23: 02: 40.2$ & -3.00 & -7.20 & 3.70 & 13.474 & 0.398 & 0.153 & 12.210 & 0.086 \\
\hline NGC 6830-9 & $19: 52: 10.97$ & $+23: 17: 06.0$ & -0.80 & -7.40 & 3.70 & 13.562 & 0.473 & 0.134 & 12.222 & 1.148 \\
\hline NGC 6830-10 & $19: 52: 25.39$ & $+22: 49: 11.6$ & 0.10 & 1.70 & 3.70 & 13.886 & 0.401 & 0.144 & 12.619 & 0.199 \\
\hline NGC 6830-11 & $19: 51: 07.65$ & $+23: 24: 32.6$ & -3.10 & -18.50 & 3.70 & 13.859 & 0.392 & 0.184 & 12.366 & 0.205 \\
\hline
\end{tabular}

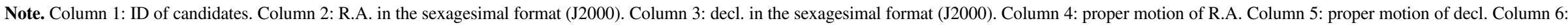

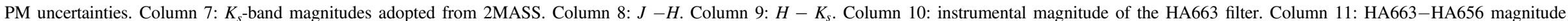
difference. 
Table 2

Observation Log and Results

\begin{tabular}{lccccc}
\hline \hline ID & Date & Telescope & Instrument & Time & R-band \\
\hline NGC 6830-1 & 2014 May 1 & Palomar 60 inch & SED Machine & $300 \mathrm{~s}$ & 11.19 \\
NGC 6830-2 & 2014 May 1 & Palomar 60 inch & SED Machine & Type \\
NGC 6830-1 & 2014 Aug 2 & Lulin 1 m & HIYOYU & Be \\
NGC 6830-1 & 2015 Jun 15 & Lick 3 m & Kast Dual Spectrograph & $600 \mathrm{~s}$ & 11.51 \\
NGC 6830-2 & 2015 Jun 15 & Lick 3 m & Kast Dual Spectrograph & $600 \mathrm{~s}$ & 11.19 \\
NGC 6830-3 & 2015 Jun 15 & Lick 3 m & Kast Dual Spectrograph & $1200 \mathrm{~s}$ & 11.19 \\
NGC 6830-4 & 2015 Jun 15 & Lick 3 m & Kast Dual Spectrograph & $1200 \mathrm{~s}$ & 13.24 \\
NGC 6830-5 & 2015 Jun 15 & Lick 3 m & Kast Dual Spectrograph & $1200 \mathrm{~s}$ & 15.28 \\
NGC 6830-6 & 2015 Jun 16 & Lick 3 m & Kast Dual Spectrograph & $1500 \mathrm{~s}$ & 14.98 \\
NGC 6830-7 & 2015 Jun 16 & Lick 3 m & Kast Dual Spectrograph & $1500 \mathrm{~s}$ & Be \\
NGC 6830-8 & 2015 Jun 15 & Lick 3 m & Kast Dual Spectrograph & $1200 \mathrm{~s}$ & 15.98 \\
NGC 6830-9 & 2015 Jun 16 & Lick 3 m & Kast Dual Spectrograph & $1500 \mathrm{~s}$ & 15.69 \\
NGC 6830-10 & 2015 Jun 16 & Lick 3 m & Kast Dual Spectrograph & $1200 \mathrm{~s}$ & 15.89 \\
NGC 6830-11 & 2015 Jun 16 & Lick 3 m & Kast Dual Spectrograph & $1500 \mathrm{~s}$ & G \\
\hline
\end{tabular}

Note. Column 1: ID of targets. Column 2: Observations dates. Column 3: Telescope name. Column 4: Instrument name. Column 5: Exposure time in seconds. Column 6: R-band magnitudes adapted from PPMXL catalog. Column 7: Spectral types.

5. Selecting possible Be stars from emission-line candidates based on the $J-H$ versus $H-K_{s}$ color-color diagram (CMD). The gray contours as shown in Figure 1(d) represent the region of 1185 known Be stars (Zhang et al. 2005). Under the assumption that most Be stars have similar infrared colors, we identified $\mathrm{Be}$ star candidates as those in NGC 6830 inside the gray-dashed region in the $\mathrm{CMD}$.

Using the above criteria, we identified 11 Be star candidates (Table 1). One candidate was confirmed as the known Be star VES 72 in the cluster NGC 6830 (listed as NGC 6830-2 in Table 1; Hoag \& Applequist 1965).

\section{SPECTROSCOPIC OBSERVATIONS AND DATA REDUCTION}

\subsection{SED-machine Observations}

To demonstrate the feasibility and capability of the SEDmachine in the study of Be stars, we obtained the optical spectra of two bright objects, VES 72 (NGC 6830-2) and a new Be star candidate NGC 6830-1 during its commission runs. Logs of observations are listed in Table 2. Due to time constraints during the commission runs, we only observed the 2 brightest candidates but not all of our 11 candidates. The SEDmachine, mounted on the Palomar 1.5-m telescope, observations were carried out on 2014 May 1 under a seeing of approximately $2^{\prime \prime}$.

The SED-machine IFU spectra were reduced independently with the pipelines developed by the NCU and the Caltech PTF team. The IFU data reduction procedures consist of the following: (1) overscan and bias subtraction, (2) automatic spectrum identification using a Xe arc-lamp spectra image, (3) calculating wavelength calibration solutions of every IFU spectra using $\mathrm{HgNeXe}$ lamp sources, (4) simple-sum extraction of all spectra, (5) reconstructing the image of the sky as seen before the prism from surrounding sky identification, and (6) object spectrum extraction with sky spectra subtraction, wavelength calibration, and flux calibration.

\subsection{Lulin 1-m Telescope (LOT) Observations}

To confirm the $\mathrm{H} \alpha$ detection from SED-machine observations, we re-observed NGC 6830-1 using the Hiyoyu spectrograph on the LOT. The LOT observations were conducted on 2014 August 2 under a seeing of approximately 1 1"5. Using a grating of $300 \mathrm{~mm}^{-1}$ and a slit width of $2^{\prime \prime}$, we covered a wavelength range of $3800-7600 \AA$. The Hiyoyu spectrum was reduced with the IRAF ${ }^{14}$ package, following the standard data reduction procedure, i.e., dark, bias-subtraced, flat-fielding-corrected, wavelength calibration, and flux calibration. The wavelength calibration was performed using a HeNeAr lamp. The flux calibration might not be very accurate due to the unstable weather during the observation.

\subsection{Lick Observations}

We used the Kast dual spectrograph on the 3-m Shane telescope at the Lick observatory to complete the confirmation of spectral typing of the $11 \mathrm{Be}$ star candidates. The observing runs were conducted on 2015 June 15-16 under a seeing of 1 ." $5-2^{\prime \prime}$ using a $2^{\prime \prime}$ slit width. Using $600 / 7500$ grating on the red side and $600 / 4310$ grism on the blue side, we can cover a wavelength range of $3500-7800 \AA$. The dispersions on the red side and blue side are 2.32 and $1.85 \AA$ pixel $^{-1}$, respectively. We reduced the spectra using the standard tools in IRAF, including corrections for overscan bias and flat-fielding using the dome flat images. We used $\mathrm{NeHg}-\mathrm{Cd}$ arc lamps for wavelength calibrations and standard stars Feige 92 and BD +284211 for flux calibration.

\section{RESULTS}

\subsection{SED-machine and LOT Spectra}

In Figure 3, we showed the SED-machine spectra of the bright Be stars NGC 6830-1 and VES 72 (NGC 6830-2). The spectrum of VES 72 shows a prominent $\mathrm{H} \alpha$ emission line and clear hydrogen and $\mathrm{He}$ I $\lambda 4471+\mathrm{Mg}$ II $\lambda 4481$ absorption lines. We further observed the new Be candidate NGC 6830-1 using

\footnotetext{
${ }^{14}$ Image Reduction and Analysis Facility. IRAF is written and supported by the National Optical Astronomy Observatories (NOAO) in Tucson, Arizona.
} 

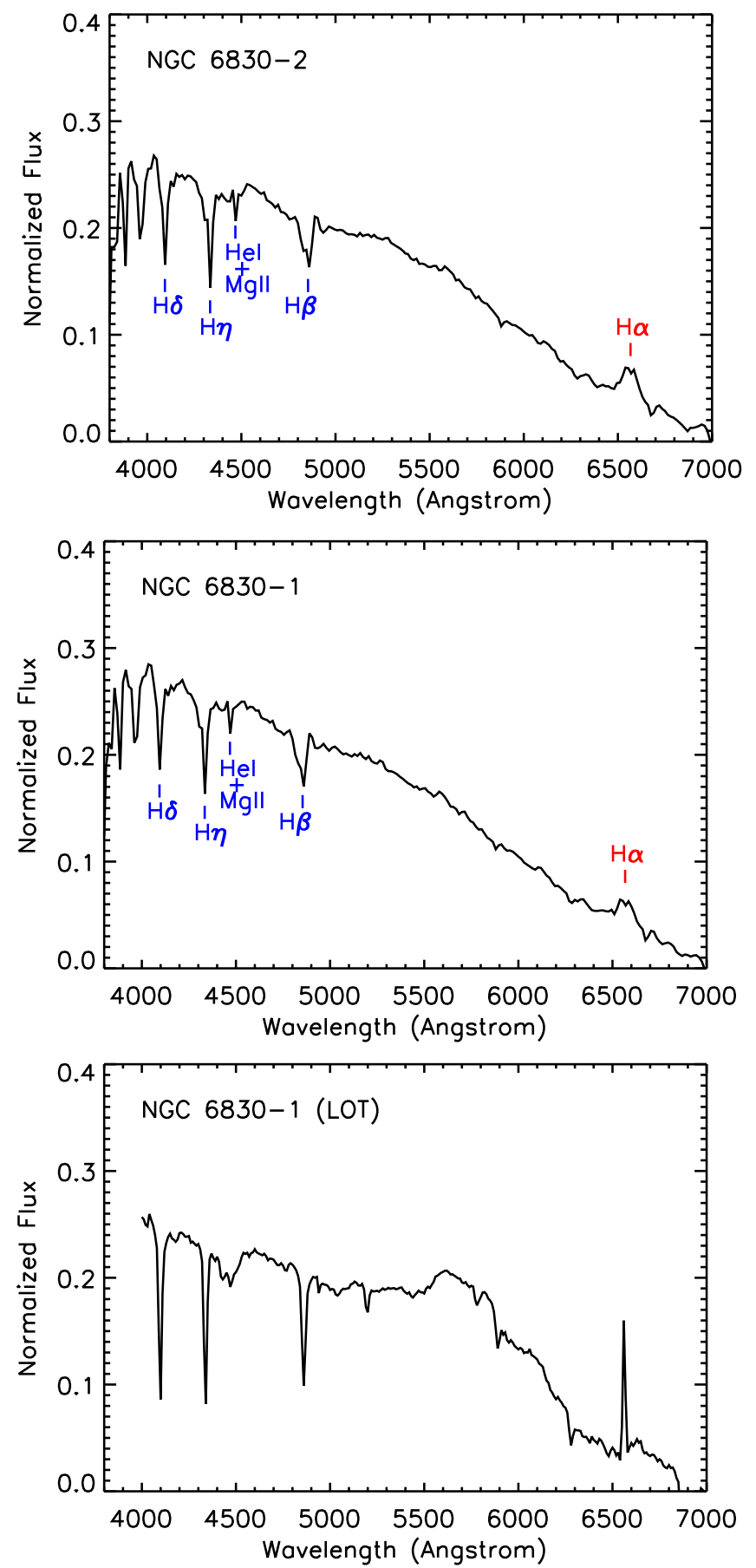

Figure 3. SED-machine results. The unit of the $y$-axis is normalized fluxes at $7000 \AA$ in logarithm. Upper panel: SED-machine spectrum of the known Be star VES 72. We detect the clear $\mathrm{H} \alpha$ emission line while other Balmer series show absorption features. Middle panel: we also detected a clear $\mathrm{H} \alpha$ emission line for the Be star candidate NGC6830-1 with the SED-machine. Lower panel: the $\mathrm{H} \alpha$ emission line also appears in the LOT spectrum for the same candidate.

the SED-machine, and the Hiyoyu spectrograph mounted on the LOT. Both SED-machine and LOT spectra present a clear detection of the $\mathrm{H} \alpha$ emission line, and hydrogen and $\mathrm{He}$ I $\lambda 4471$ $+\operatorname{Mg}$ II $\lambda 4481$ absorption lines, indicating a Be-type. With a spectral resolution of $\lambda / \delta \lambda \sim 333$ of the Hiyoyu spectragraph, we estimated an equivalent width of the $\mathrm{H} \alpha$ emission-line, EW $[\mathrm{H} \alpha]=-5 \AA$ for NGC 6830-1. Although the spectral resolution is too poor to classify the spectral subtype, the similar brightness of NGC 6830-1 to VES 72 suggests similar subtypes (e.g., B6-type).

\subsection{Lick Spectra}

We present the spectra of Be star candidates obtained by the Lick $3 \mathrm{~m}$ Shane telescope in Figure 4. Only three Be star candidates (NGC6830-1, NGC6830-2, and NGC6830-3) show $\mathrm{H} \alpha$ emission and B-type spectra. All candidates are discussed in the following subsections.

\subsubsection{Confirmed Be Stars}

1. NGC 6830-1: this candidate shows a prominent $\mathrm{H} \alpha$ emission line with the $\mathrm{EW}[\mathrm{H} \alpha]$ of $-7 \AA$ and hydrogen absorption lines of $\mathrm{H} \beta, \mathrm{H} \gamma, \mathrm{H} \epsilon$, and $\mathrm{H} \delta$. The $\mathrm{EW}[\mathrm{H} \alpha]$ is similar to that of the LOT observation. It also shows $\mathrm{He}_{\mathrm{I}} \lambda 4026$, He I $\lambda 4387$, and $\mathrm{He}_{\mathrm{I}} \lambda 4471+\mathrm{Mg}$ II $\lambda 4481$ absorption lines, the features of B-type stars. We thus confirmed the candidate as a newly discovered Be star.

2. NGC 6830-2: NGC6830 2 is a known Be star VES 72, a B6-type star with photoelectric observations (Hoag \& Applequist 1965). We also detected the $\mathrm{H} \alpha$ emission line, with the EW $[\mathrm{H} \alpha]=-9 \AA$. It shows similar hydrogen and helium/magnesium absorptions lines as in NGC 6830-1.

3. NGC 6830-3: the star shows the $\mathrm{H} \alpha$ line in emission with the $\mathrm{EW}[\mathrm{H} \alpha]=-8 \AA$. In addition to the same hydrogen and helium/magnesium absorption lines detected in NGC 6830-1 and NGC 6830-2, it also shows the He I $\lambda 4009$ absorption line. We thus classified the star as a Be star. The star is 2 mag fainter than NGC 6830-1 and NGC 6830-2, implying a late-type B9 star.

\subsubsection{Non-Be Star Candidates}

1. NGC 6830-4: we do not detect the $\mathrm{H} \alpha$ emission line for the star, and it shows the $\mathrm{Ca}$ II $\mathrm{K}$ absorption line, indicating the star might be a late-A or F-type star.

2. NGC 6830-5: it shows not only the $\mathrm{Ca}$ II $\mathrm{K}$ absorption line, but also the G-band absorption at $4300 \AA$. The star is classified as a G-type star.

3. NGC 6830-6-NGC 6830-11: compared to NGC 6830-5, these stars show stronger G-band absorption, and weaker $\mathrm{H} \gamma$ and $\mathrm{H} \epsilon$ absorption lines. We classify NGC 6830-6NGC 6830-11 as G-type stars.

\subsection{Infrared Color}

The Be star NGC 6830-3 shows an infrared excess $H-K_{s}=0.316$ (Figure $1(\mathrm{~d})$ ), as in many known Be stars. Such infrared excess can be explained by free-free emission from circumstellar disks (Lee \& Chen 2011). For comparison, we included the fluxes of $3.4 \mu \mathrm{m}$ (W1), $4.6 \mu \mathrm{m}$ (W2), $12 \mu \mathrm{m}$ (W3), and $22 \mu \mathrm{m}$ (W4) from the Wide-field Infrared Survey Explorer (WISE; Wright et al. 2010), and plotted in Figure 5 the spectral energy distribution between 0.4 and $25 \mu \mathrm{m}$ of three Be stars (Table 3). All of the objects have no significant $22 \mu \mathrm{m}$ detection, suggesting no presence of warm dust. Although the detection is only an upper limit, it seems that NGC 6830-3 might have a different W3-W4 color from NGC 6830-1 and NGC 6830-2. Observations with high resolution and sensitivity are in demand to confirm the $22 \mu \mathrm{m}$ detection in NGC 6830-3 in the future. 


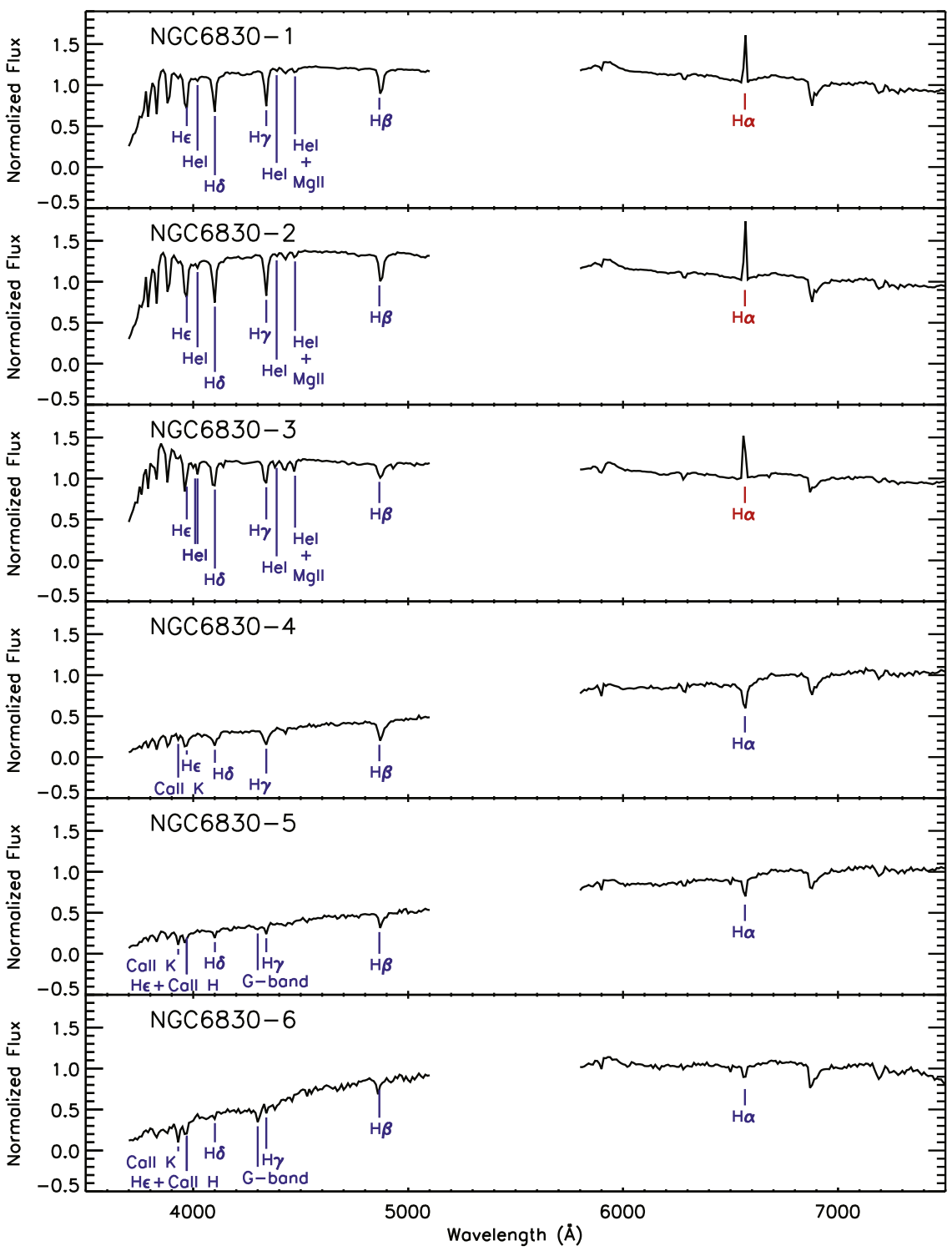

Figure 4. Lick spectra of Be star candidates. Fluxes are normalized at $7000 \AA$.

\subsection{Optical Variability}

Be stars are also known as photometric and spectroscopic variables. Their variabilities show a wide range of timescales from much less than a day (Percy et al. 2002) to more than several decades (Okazaki 1997). Compare to normal B-type stars, photometric irregular variation is frequently seen in $\mathrm{Be}$ stars (Kourniotis et al. 2014). There are three types of variability found in Be stars: (1) short-term variability with timescales of days (Hubert et al. 1997); (2) mid-term variability with timescales of days and weeks with the amplitude of 0.3 mag (Okazaki 1997; Hubert \& Floquet 1998); (3) long-term variability with timescales of years and larger amplitudes of $>0.3 \mathrm{mag}$ (Hubert \& Floquet 1998). Since the PTF R-band light curve data saturated around the 14th magnitude, we adopted the V-band light curve data from the All Sky Automated Survey (ASAS; Pojmañski 2002) to investigate the variability of our detected Be stars. The ASAS is a project to monitor stars brighter than $14 \mathrm{mag}$ to investigate their photometric variability; thus it is suitable to search for longterm variability with large amplitude $>0.3$ mag for Be stars.
We chose two stars, star1 and star2 in the same field, as the photometric references to build up the light curves using the differential photometry technique. We only used the data with grade A from the ASAS catalog, i.e., the best data with photometric uncertainties $<0.05 \mathrm{mag}$ in the catalog. The value $\sigma_{\mathrm{sc}}$ was defined as the standard deviation of magnitude difference between the reference and the Be stars, and used to determine the significance of the variability. The $\sigma_{\mathrm{sc}}$ against star1 and star2 of NGC6830-1, NGC6830-2, and NGC6830-3 are 0.15 and $0.14,0.14$, and 0.16 , and 0.26 and $0.23 \mathrm{mag}$, respectively (Figure 6). These $\sigma_{\mathrm{sc}}$ values are all comparable to the systematic errors of corresponding magnitudes in ASAS (Pojmañski 2002). Therefore, we do not detect any significant long-term variability in the optical band for these three Be stars.

\section{SUMMARY AND DISCUSSION}

In summary, we apply PTF's $\mathrm{H} \alpha$ imaging photometry to identify $11 \mathrm{Be}$ star candidates in NGC 6830. Three stars have been confirmed as $\mathrm{Be}$ stars with intermediate and late-type spectra by using the SED-machine on the Palomar $1.5 \mathrm{~m}$ 


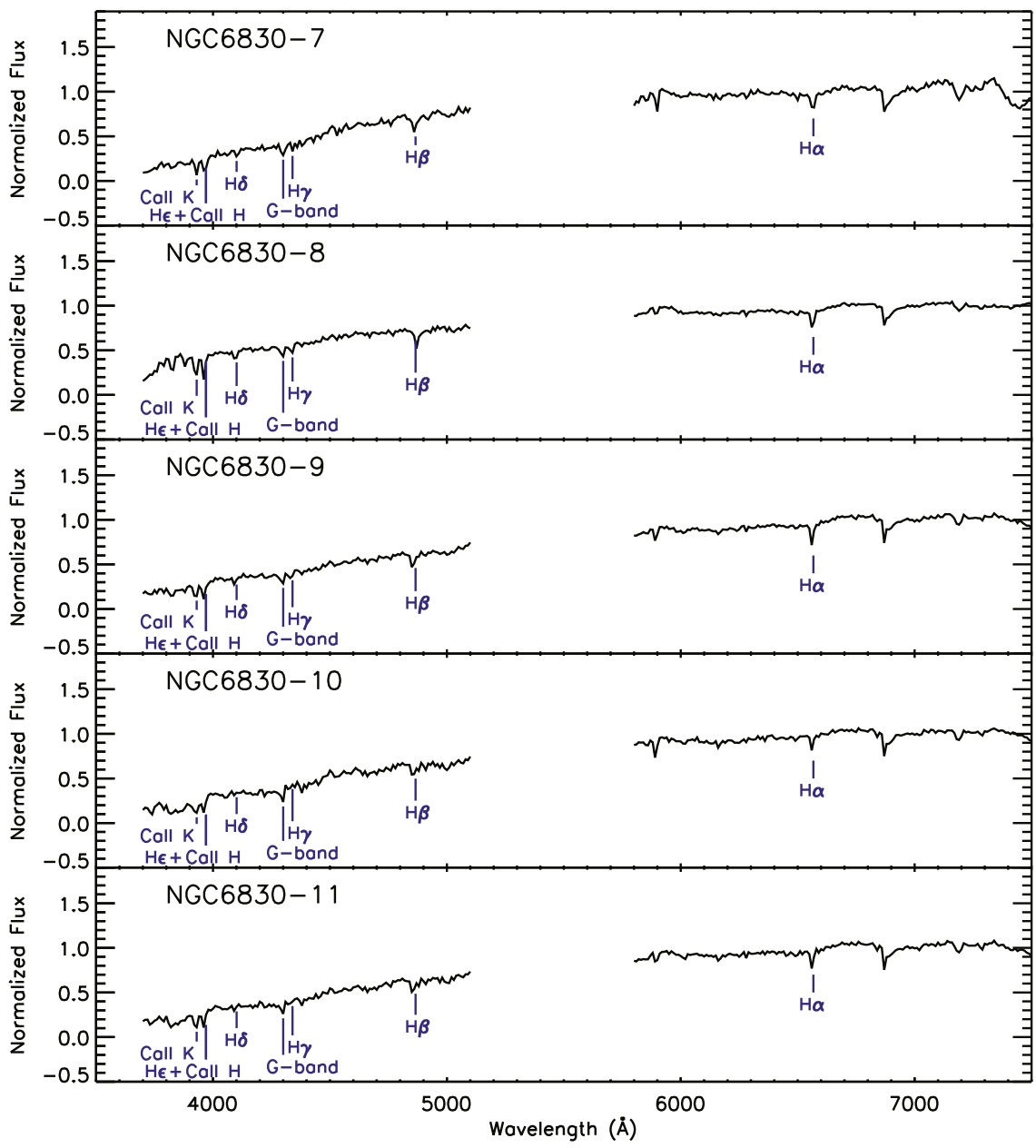

Figure 4. (Continued.)

telescope, the Hiyoyu spectrograph on the Lulin 1-m telescope, and the Kast dual spectrograph on the Lick 3-m telescope. We suggest that there are three Be stars in the cluster NGC 6830. The spatial distribution of the Be stars might be caused by gravitational disruption over time. We also present the results of the SED-machine; this study demonstrated that the high efficiency of the SED-machine can provide rapid observations for Be stars in a comprehensive survey in the future.

We suggest that there are three $\mathrm{Be}$ stars in the cluster NGC 6830. McSwain \& Gies (2005) studied the fraction of Be stars in eight clusters with an age older than $100 \mathrm{Myr}$; only four clusters have up to two Be stars. These results indicate that old open clusters lack Be stars. Although our results are consistent with the previous conclusions, it should be noted that either the relaxation process or Galactic external perturbation (Chen et al. 2004) might have happened in open clusters. As shown in Figure 7, the Be star NGC6830-3 is located in the outer region of the cluster, while NGC6830-1 and NGC6830-2 are located in the central region. With an age of $125 \mathrm{Mys}$, the open cluster NGC 6830 might have a very irregular shape and become loose. The spatial distribution of Be stars could be the consequence of the relaxation process. Since McSwain \& Gies (2005) searched Be stars within a fixed angular size, more Be stars could be discovered if a larger size is used.
Dachs et al. (1988) proposed an empirical relation between the disk fraction $f_{D}$ and the $\mathrm{EW}[\mathrm{H} \alpha]$ for Be stars:

$$
f_{D} \simeq 0.1 \times \frac{\mathrm{EW}[\mathrm{H} \alpha]}{-30 \AA},
$$

where $f_{D}$ is the ratio of disk radiation to total radiation of the star-disk system. The EW $[\mathrm{H} \alpha]$ of the Be stars in NGC 6830 are all greater than $-10 \AA$, suggesting the disk fraction $f_{D}<0.03$. The result is consistent with Dachs et al. (1988), who showed that most late-type Be stars (B4-B7) have $f_{D}<0.1$, while most early-type Be stars (B1-B3) have $f_{D}>0.1$.

Several mechanisms might cause long-term variability in $\mathrm{Be}$ stars: (1) long-lived strong outbursts (Hubert \& Floquet 1998), (2) the decline of brightness due to the cooling envelope (Koubský et al. 1997), and (3) a weak brightness change related to $\mathrm{H} \alpha$ variability. The brightness of NGC 6830-1 and NGC 6830-2 allow us to investigate the variability with the amplitude larger than 0.15 mag using the ASAS data set. We do not discover any significant variability for NGC 6830-1 and NGC 6830-2. This is consistent with the previous studies; Hubert \& Floquet (1998) showed that only 12\% of B6-type Be stars have the variability with the amplitude of $0.12-0.3 \mathrm{mag}$. Because large amplitude caused by strong outbursts is usually 

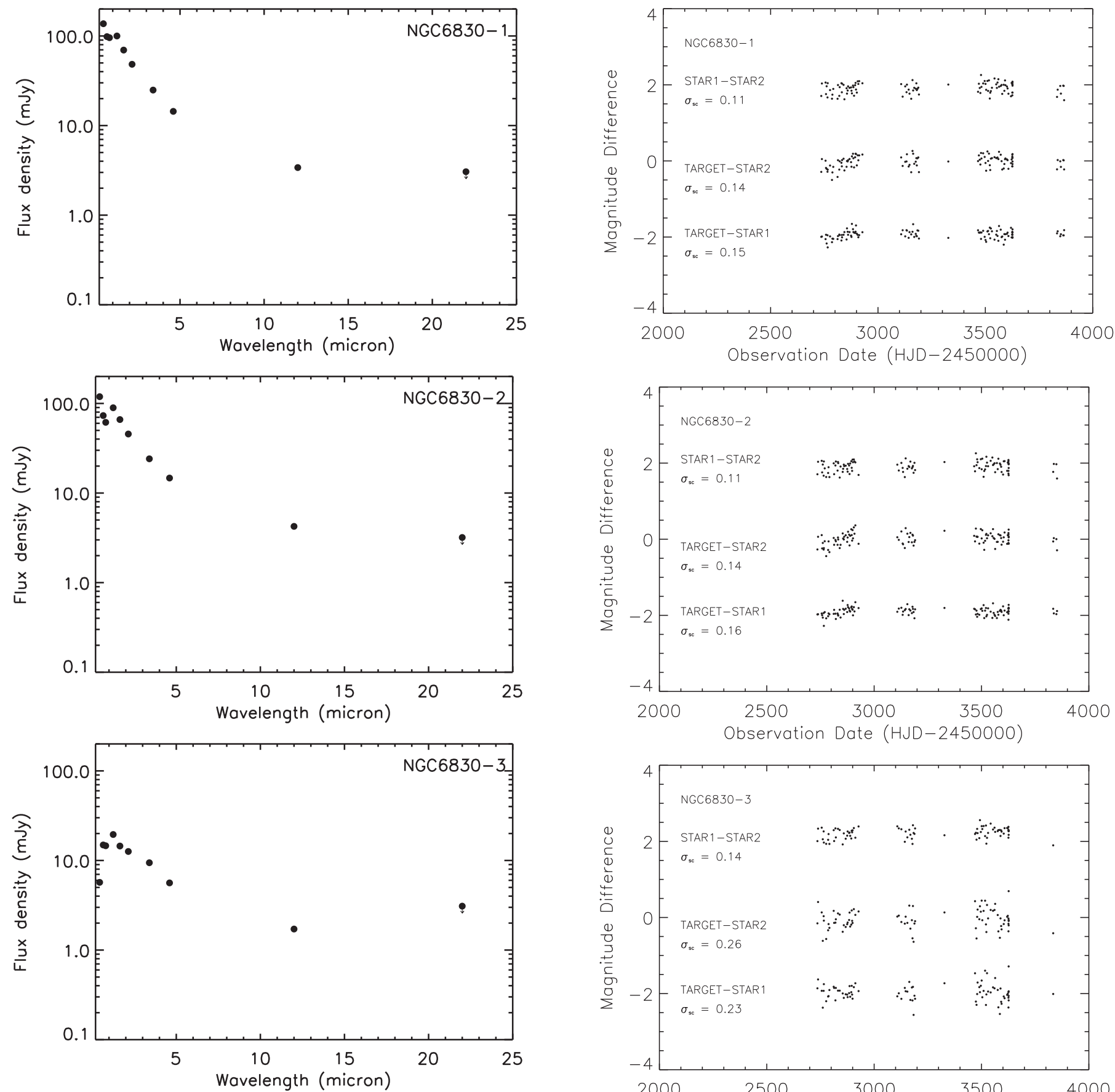

Figure 5. Spectral energy distribution between 0.4 and $25 \mu \mathrm{m}$. Detections at $22 \mu \mathrm{m}$ for these Be stars are upper limits. Flux densities of 445, 658, and $806 \mathrm{~nm}$ are adopted from PPMXL; flux densities of $1.25,1.65$, and $2.15 \mu \mathrm{m}$ are adopted from 2MASS; 3.4, 4.6, 12, and $22 \mu \mathrm{m}$ flux densities are adopted
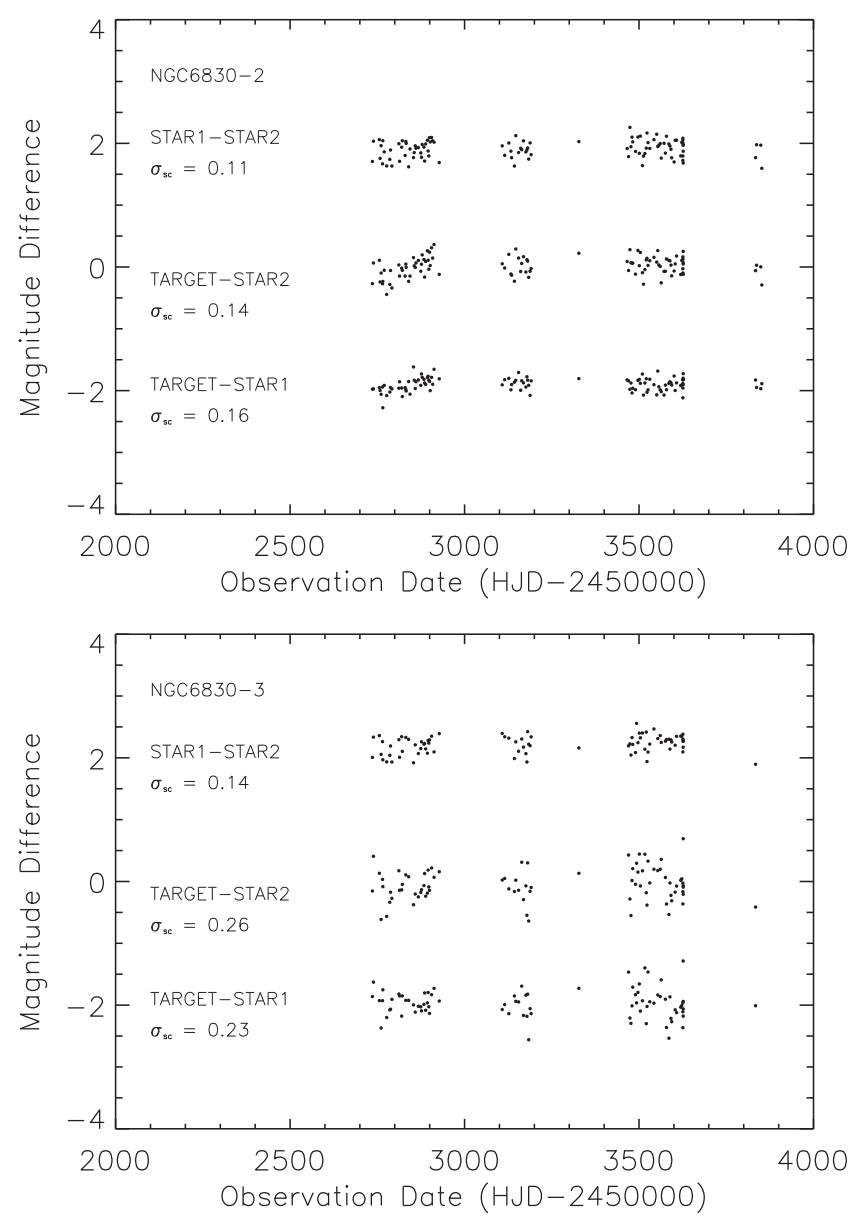

Figure 6. Differential photometry of Be stars in NGC 6830. from WISE.

Table 3

Flux Density of Be Stars in NGC 6830

\begin{tabular}{lcccccccccc}
\hline \hline ID & $\begin{array}{c}0.445 \mu \mathrm{m} \\
(\mathrm{mJy})\end{array}$ & $\begin{array}{c}0.658 \mu \mathrm{m} \\
(\mathrm{mJy})\end{array}$ & $\begin{array}{c}0.806 \mu \mathrm{m} \\
(\mathrm{mJy})\end{array}$ & $\begin{array}{c}1.25 \mu \mathrm{m} \\
(\mathrm{mJy})\end{array}$ & $\begin{array}{c}1.65 \mu \mathrm{m} \\
(\mathrm{mJy})\end{array}$ & $\begin{array}{c}2.2 \mu \mathrm{m} \\
(\mathrm{mJy})\end{array}$ & $\begin{array}{c}3.4 \mu \mathrm{m} \\
(\mathrm{mJy})\end{array}$ & $\begin{array}{c}4.6 \mu \mathrm{m} \\
(\mathrm{mJy})\end{array}$ & $\begin{array}{c}12 \mu \mathrm{m} \\
(\mathrm{mJy})\end{array}$ & $\begin{array}{c}22 \mu \mathrm{m} \\
(\mathrm{mJy})\end{array}$ \\
\hline NGC 6830-1 & 137 & 98.3 & 95.7 & 100 & 69.6 & 48.3 & 24.9 & 14.4 & 3.397 & 3.053 \\
NGC 6830-2 & 119 & 73.2 & 61.5 & 89.5 & 66.1 & 45.6 & 24.1 & 14.7 & 4.245 \\
NGC 6830-3 & 5.7 & 14.9 & 14.6 & 19.5 & 14.5 & 12.6 & 9.434 & 5.621 & 1.717 & 3.093 \\
\hline
\end{tabular}

Note. Column 1: ID of targets. Column 2 to 11: Flux density in millijansky adopted from the PPMXL, 2MASS, and WISE catalogs. The detections at $22 \mu \mathrm{m}$ for three Be stars are upper limits. 


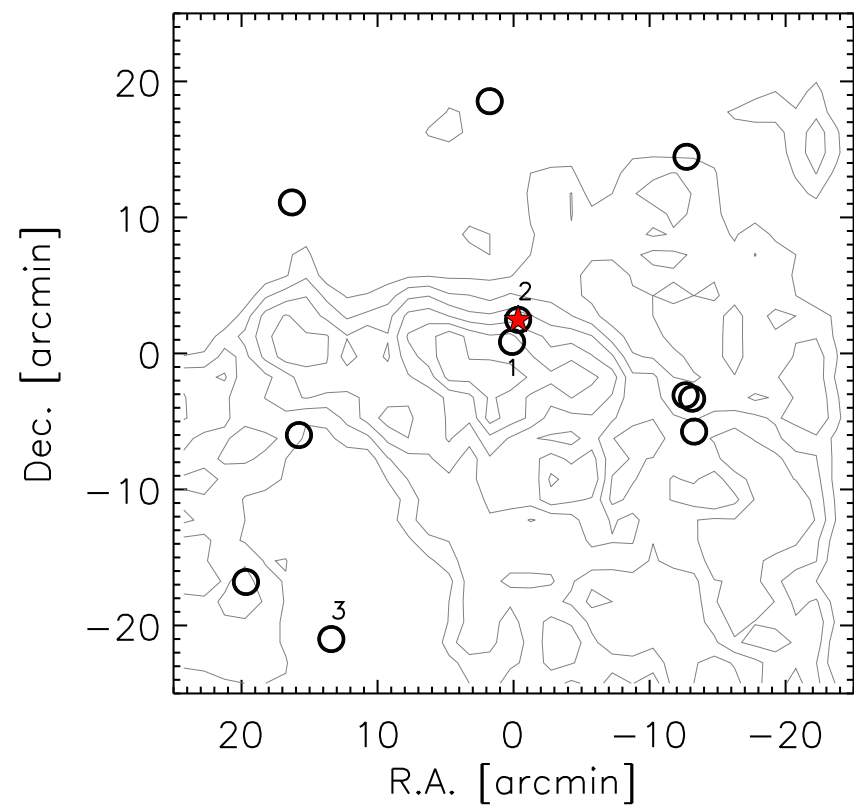

Figure 7. Spatial distribution of Be star candidates. The open circles: Be star candidates; red pentagram: the known Be star VES 72. Contours represent the distribution of stars within a field of $50^{\prime} \times 50^{\prime}$.

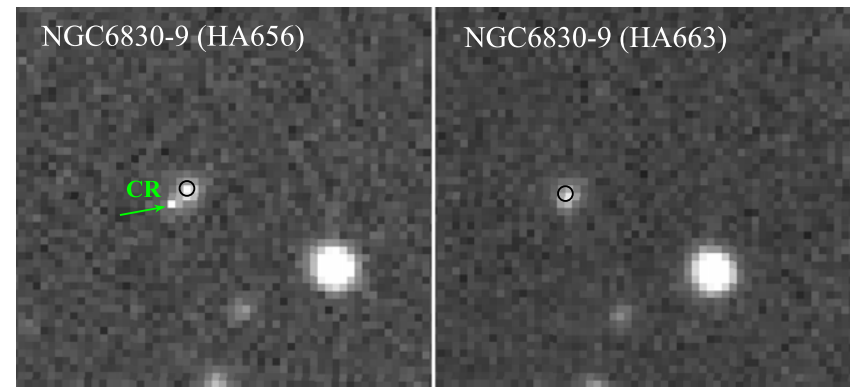

Figure 8. HA656 (left) and HA663 (right) images of NGC6830-9. The green arrow indicates the residual cosmic-ray. Black open circles represent the position of NGC 6830-9.

seen in the earliest Be stars, it is not surprising that we do not detect variability in these Be stars.

Finally, as a pilot project to search for Be stars in clusters with different ages, we reviewed our selection criteria for NGC 663 (Yu et al. 2015) and in this work. Of the $11 \mathrm{Be}$ candidates, 8 are classified as A- or G-type stars; most of them have marginal $\mathrm{H} \alpha$ detection in the HA663-HA656 color diagram (Figure 2). This misidentification can be improved by using the 2MASS color-color diagram. As shown in Figure 1(d), if we cause the selection region (dashed line) to have a similar range as the known Be stars, most G-type stars would have been ruled out. Thus, we will change our selection criteria in our future project. Moreover, it should be noted that the star NGC6830-9 has a large $\mathrm{H} \alpha$ excess in Figure 2, while the optical spectrum suggested a G-type star with the $\mathrm{H} \alpha$ absorption line. This is caused by the contamination of a residual cosmic-ray hitting on the target region, which is not easy to eliminate. Procedures for removing cosmic-rays around science targets will be performed carefully in a future project.

We thank the referee for constructive comments. We are also grateful to the staff of Lulin and Lick Observatory for helping with the observations. This work is supported, in part, by the
National Science Council and the Ministry of Science and Technology of Taiwan under grants MOST 104-2119-M-008024 (W.-H.I.), MOST 103-2112-M-008-024-MY3 (W.-P.C.), MOST 104-2112-M-008-012-MY3 (C.-C.N.), and NSC 1032917-I-564-004 (P.-C.Y.). The Lulin Observatory is funded by the Ministry of Science and Technology of Taiwan, and operated by National Central University of Taiwan. The Lick Observatory is funded by Google Inc. and operated by the University of California. This publication makes use of data products from the Two Micron All Sky Survey, which is a joint project of the University of Massachusetts and the Infrared Processing and Analysis Center/California Institute of Technology, funded by the National Aeronautics and Space Administration and the National Science Foundation. This publication makes use of data products from the Wide-field Infrared Survey Explorer, which is a joint project of the University of California, Los Angeles, and the Jet Propulsion Laboratory/California Institute of Technology, funded by the National Aeronautics and Space Administration.

\section{REFERENCES}

Barkhatova, K. A. 1957, SvA, 1, 822

Ben-Ami, S., Konidaris, N., Quimby, R., et al. 2012, Proc. SPIE, 8446, 86 Carciofi, A. C., \& Bjorkman, J. E. 2006, ApJ, 639, 1081

Carciofi, A. C., Okazaki, A. T., le Bouquin, J.-B., et al. 2009, A\&A, 504, 915 Chen, W. P., Chen, C. W., \& Shu, C. G. 2004, AJ, 128, 2306

Chojnowski, S. D., Whelan, D. G., Wisniewski, J. P., et al. 2015, AJ, 149, 7 Cutri, R. M., Skrutskie, M. F., van Dyk, S., et al. 2003, yCat, 2246, 0

Dachs, J., Kiehling, R., \& Engels, D. 1988, A\&A, 194, 167

de Mink, S. E., Langer, N., Izzard, R. G., Sana, H., \& de Koter, A. 2013, ApJ, 764,166

Drew, J. E., Greimel, R., Irwin, M. J., et al. 2005, MNRAS, 362, 753

Fabregat, J., \& Torrejón, J. M. 2000, A\&A, 357, 451

Girardi, L., Bertelli, G., Bressan, A., et al. 2002, A\&A, 391, 195

Hoag, A. A., \& Applequist, N. L. 1965, ApJS, 12, 215

Hubert, A. M., \& Floquet, M. 1998, A\&A, 335, 565

Hubert, A. M., Floquet, M., Hao, J. X., et al. 1997, A\&A, 324, 929

Jaschek, C., Jaschek, M., \& Kucewicz, B. 1964, ZA, 59, 108

Keller, S. C., Grebel, E. K., Miller, G. J., \& Yoss, K. M. 2001, AJ, 122, 248

Kharchenko, N. V., Piskunov, A. E., Schilbach, E., Röser, S., \& Scholz, R.-D. 2012, A\&A, 543, 156

Kharchenko, N. V., Piskunov, A. E., Schilbach, E., Röser, S., \& Scholz, R.-D. 2013, A\&A, 558, A53

Koubský, P., Harmance, P., Kubát, J., et al. 1997, A\&A, 328, 551

Kourniotis, M., Bonanos, A. Z., Soszyński, I., et al. 2014, A\&A, 562, 125

Kraus, S., Monnier, J. D., Che, X., et al. 2012, ApJ, 744, 19

Laher, R. R., Surace, J., Grillmair, C. J., et al. 2014, PASP, 126, 674

Law, N. M., Kulkarni, S. R., Dekany, R. G., et al. 2009, PASP, 121, L1395

Lee, C.-D., \& Chen, W.-P. 2011, in IAU Symp. 272, Active OB stars: structure, evolution, mass loss, and critical limit, ed. C. Neiner, G. Wade, G. Meynet, \& G. Peters (Cambridge: Cambridge Univ. Press), 366

Lee, U., Osaki, Y., \& Saio, H. 1991, MNRAS, 250, 432

Lin, C. C., Hou, J. L., Chen, L., et al. 2015, RAA, 15, 8

Martayan, C., Baade, D., \& Fabregat, J. 2010, A\&A, 509, 11

Mathew, B., \& Subramaniam, A. 2011, BASI, 39, 517

Mathew, B., Subramaniam, A., \& Bhatt, B. C. 2008, MNRAS, 388, 1879

Mclaughlin, D. B. 1932, POMic, 4, 175

McSwain, M. V., \& Gies, D. R. 2005, ApJS, 161, 118

McSwain, M. V., Huang, W., \& Gies, D. R. 2009, ApJ, 700, 1216

Meilland, A., Millour, F., Kanaan, S., et al. 2012, A\&A, 538, 110

Ngeow, C. C., Konidaris, N., Quimby, R., et al. 2013, in IAU Symp. 290, Feeding Compact Objects: Accretion on All Scales, ed. C. M. Zhang, T. Belloni, M. Méndez, \& S. N. Zhang (Cambridge: Cambridge Univ. Press), 281

Okazaki, A. T. 1997, A\&A, 318, 548

Peña, J. H., Machado, L. F., Garcia, H., et al. 2011, RMxAA, 47, 309 Percy, J. R., Hosick, J., Kincaide, H., \& Pang, C. 2002, PASP, 114, 551 Pojmañski, G. 2002, AcA, 52, 397

Pols, O. R., Cotẽ, J., Waters, L. B. F. M., \& Heise, J. 1991, A\&A, 241, 419 Porter, J. M., \& Rivinius, T. 2003, PASP, 115, 1153

Quirrenbach, A., Bjorkman, K. S., Bjorkman, J. E., et al. 1997, ApJ, 479, 477 
Quirrenbach, A., Buscher, D. F., Mozurkewich, D., Hummel, C. A., \& Armstrong, J. T. 1994, A\&A, 283, L13

Raddi, R., Drew, J. E., Steeghs, D., et al. 2015, MNRAS, 446, 274

Ritter, A., Ngeow, C. C., Konidaris, N., Quimby, R., \& Ben-Ami, S. 2014, CoSka, 43, 209

Rivinius, T., Baade, D., \& Štefl, S. 2003, A\&A, 411, 229

Rivinius, T., Carciofi, A. C., \& Martayan, C. 2013, A\&ARv, 21, 69

Röser, S., Demleitner, M., \& Schilbach, E. 2010, AJ, 139, 2440
Shao, Y., \& Li, X. D. 2014, ApJ, 796, 37

Townsend, R. H. D., Owocki, S. P., \& Howarth, I. D. 2004, MNRAS, 350, 189

Wisniewski, J. P., \& Bjorkman, K. S. 2006, ApJ, 652, 458

Wood, K., Bjorkman, K. S., \& Bjorkman, J. E. 1997, ApJ, 477, 926

Wright, E. L., Eisenhardt, P. R. M., Mainzer, A. K., et al. 2010, AJ, 140,1868

Yu, P.-C., Lin, C. C., Chen, W. P., et al. 2015, AJ, 149, 43

Zhang, P., Chen, P. S., \& Yang, H. T. 2005, NewA, 10, 325 